\title{
The Impact of GWAS Findings on Cancer Etiology and Prevention
}

\author{
Jane C. Figueiredo • Daniel O. Stram • \\ Christopher A. Haiman
}

Published online: 3 July 2014

(C) Springer International Publishing AG 2014

\begin{abstract}
Genome-wide association studies (GWAS) of common genetic variation have contributed immensely to our understanding of inherited susceptibility to cancer. To date, over 400 susceptibility loci have been identified across all cancers. These loci implicate novel as well as established genes and biological pathways, and have reinforced the significance and functionality of non-proteincoding DNA sequence. While the genetic associations for each variant are deemed modest, for some cancers, risk stratification based on aggregate effects may be of value for targeted screening and prevention strategies. Several questions still remain to be answered in order to fully assess the significance of these findings, including a better understanding of the source of the missing heritability. Here we discuss, from studies of common cancers, the impact of GWAS findings on illuminating disease etiology, the potential future utility of polygenic models for screening and prevention, and the future of association studies in the post-genomic era.
\end{abstract}

Keywords GWAS · Cancer · Prostate · Breast - Colorectal · Etiology $\cdot$ Prevention

\section{Introduction}

The late 2000s marked the first publications of genome-wide association studies (GWAS) in cancer, with the discovery of a limited number of loci for the most common malignancies, including the 8q24 region in colorectal, prostate, and breast cancers [1-3]. Since then, as for other complex diseases, there

J. C. Figueiredo • D. O. Stram • C. A. Haiman $(\bowtie)$

Department of Preventive Medicine, Keck School of Medicine,

University of Southern California, Los Angeles, CA, USA

e-mail: Christopher.Haiman@med.usc.edu has been impressive and unprecedented growth in the number of identified common susceptibility loci [4]. Currently, individual studies and meta-analyses have enumerated approximately 40 susceptibility loci for colorectal cancer, $>70$ for breast cancer, and $>100$ for prostate cancer [4]. These findings have helped to confirm the importance of polygenic inheritance resulting from multiple low-penetrant alleles in cancer. It is estimated that these loci may explain approximately $18 \%$ of the familial component (i.e. the risk associated with a positive family history) of breast cancer [5•] and $30 \%$ of the familial component of prostate cancer [6•]. While this is encouraging, there remains a large fraction of missing heritability that has yet to be explained for most common cancers [7•]. Whether this missing heritability is due to large numbers of common and/or rare alleles is currently not known; however, both are likely, with gains in evidence for both requiring even larger consortia studies and the incorporation of large-scale nextgeneration sequencing as the primary platform for providing data for variant identification and testing.

Inarguably, one of the primary goals of GWAS, relative to the preceding single-variant/candidate-gene approaches, has been to take an unbiased and agnostic view to uncover the genes and biological pathways that contribute (unambiguously) to cancer development. Second has been the expectation that common susceptibility alleles, alone or in combination, would improve population risk stratification and prediction models with family history and/or other previously established risk factors, thereby improving the effectiveness of screening and prevention efforts. In this review, we discuss the success and limitations of GWAS with regard to these goals, with a particular focus on the most common cancers of the breast, prostate, and colorectum, where a large number of risk loci have been revealed (because of available samples in consortia efforts), and the potential utility of risk stratification assessed in a clinical context. 


\section{Impact on Cancer Etiology}

Importance of Non-protein-Coding Sequence

An important observation is that the vast majority of risk variants (and their proxies) have been located in nonprotein-coding sequence, which points to the functional nature of non-coding DNA sequence (as highlighted by ENCODE $[8 \cdot \bullet$ ] and the National Institutes of Health Roadmap Epigenomics Project) and gene regulation rather than protein modification underlying the genetic effects. The etiologic mechanisms at non-coding susceptibility loci have also been informed through the incorporation of epigenetic information (i.e., chromatin histone modifications). For example, functional characterization of 71 breast cancer susceptibility loci showed that of 1,005 single nucleotide polymorphisms (SNPs) in linkage disequilibrium (LD) with the index SNPs $\left(r^{2} \geq 0.5\right)$, only 21 were in exons (and only two predicted as non-benign coding variants); in contrast, there were 76 SNPs in predicted transcription start-site regions and 921 SNPs in putative enhancers at 60 of the 71 breast cancer risk loci [9]. Similarly, for an investigation of 77 prostate cancer susceptibility loci, a seemingly large number of variants or their proxies in high LD were in non-coding regions but putatively functional variants, 509 SNPs located in enhancers and 20 SNPs in promoters, compared with a relative handful of coding variants [10]. In addition to influencing regulation of genes, there is also growing evidence for the importance of long non-coding RNA $[11,12]$.

\section{Highlighting Known and Novel Genes and Biological Pathways}

Several risk variants are located within or in close proximity to genes in pathways previously implicated in cancer, which has helped to reinforce and clarify biological understanding of these pathways in cancer development. For example, in colorectal cancer, the transforming growth factor- $\beta$ (TGF $\beta$ ) signaling pathway plays a critical role in proliferation, differentiation, cell migration, adhesion, and extracellular matrix production, which are key processes in development and progression of tumors $[13,14]$. Of the 40 regions identified to date through GWAS, six loci implicate genes involved in TGF $\beta$ signaling (Fig. 1), including (rs4939827) SMAD7 [15, 16], (rs4779584) GREM1 [17], (rs10411210) RHPN2, the bone morphogenetic protein genes (rs961253) BMP2 and (rs4444235) BMP4 [15], and (rs4925386) LAMA5 [18], which is required for the production of noggin, a secreted BMP antagonist [19]. Similar corollaries have been noted in breast cancer, with susceptibility loci containing genes involved in mammary development (FGFR2, PTHLH, TBX3), DNA repair (e.g., BRCA2, RAD51B, and MERIT40), hormone signaling (ESR1, NRIP1), and cell cycle regulation (CDKN2A,
$C D K N 2 B, C C N D 1$ ) [20]. In prostate cancer, prominent examples include $K L K 3$ (PSA), the androgen receptor, and $M S M B$ ( $\beta$-microseminoprotein [MSP]) [21], which is one of the most highly secreted proteins from the prostate and a previously hypothesized biomarker of prostate cancer detection or risk. Laboratory and population-based studies have demonstrated that the risk allele (rs10993994) at the MSMB locus influences CREB binding and is associated with lower MSMB gene expression in tumor cell lines and prostate tissue [22, 23], as well as lower circulating MSP levels in the blood [24]. We have also shown MSP levels to be an independent and highly significant risk factor for prostate cancer in multiple populations, a study that was motivated by the GWAS discovery at this locus [25].

It is important to note that for most of these risk loci, the presumed "affected" genes are implicated on the basis of proximity to the index variant, whereas it is possible that the relevant gene is not in the immediate vicinity. With this caveat in mind, what is interesting is that a number of risk loci harbor genes where the previous link with cancer has been weak. For example, in breast cancer, rs3803662 is just upstream of the gene TNRC9 (TOX3), a high mobility group box family member, which has been implicated in T-cell development in the thymus. The TOX gene family contains a high mobility group box motif, which is found in many eukaryotic proteins that are involved in bending and unwinding of DNA, suggesting a role in chromatin assembly. How a gene involved in Tcell development and chromatin assembly is related to breast cancer carcinogenesis is not entirely known. This is one such example of how GWAS findings may lead to hypotheses to examine new biological links with cancer.

\section{Pleiotropy in Cancer}

GWAS have also revealed a shared genetic underpinning for a number of cancers (Fig. 2). Although they yet lack validation, these pleiotropic associations [26], defined as a single variant or multiple variants in the same region of the genome being associated with risk of different cancers, indicate shared biological pathways of importance, which is perhaps not entirely surprising given our hypothesis that cancers generally evolve from a common sequence of events (e.g., uncontrolled cell proliferation and altered DNA repair capacity). Examples include genetic variants at chromosome 8q24, which have been associated with prostate, colorectum, breast, bladder, and other cancers $[3,27,28]$. Similarly, genetic variants in and near the telomerase reverse transcriptase (TERT) gene at $5 \mathrm{p} 15$, which encodes for telomerase activity, have been associated with glioma, lung, prostate, colorectal, breast, and other cancers $[18,29,30]$, emphasizing the importance of cellular aging in cancer development. A third example is the cyclin D1 locus at $11 \mathrm{q} 13$, which is a gene that is involved in regulating cell cycle progression and has been associated with 


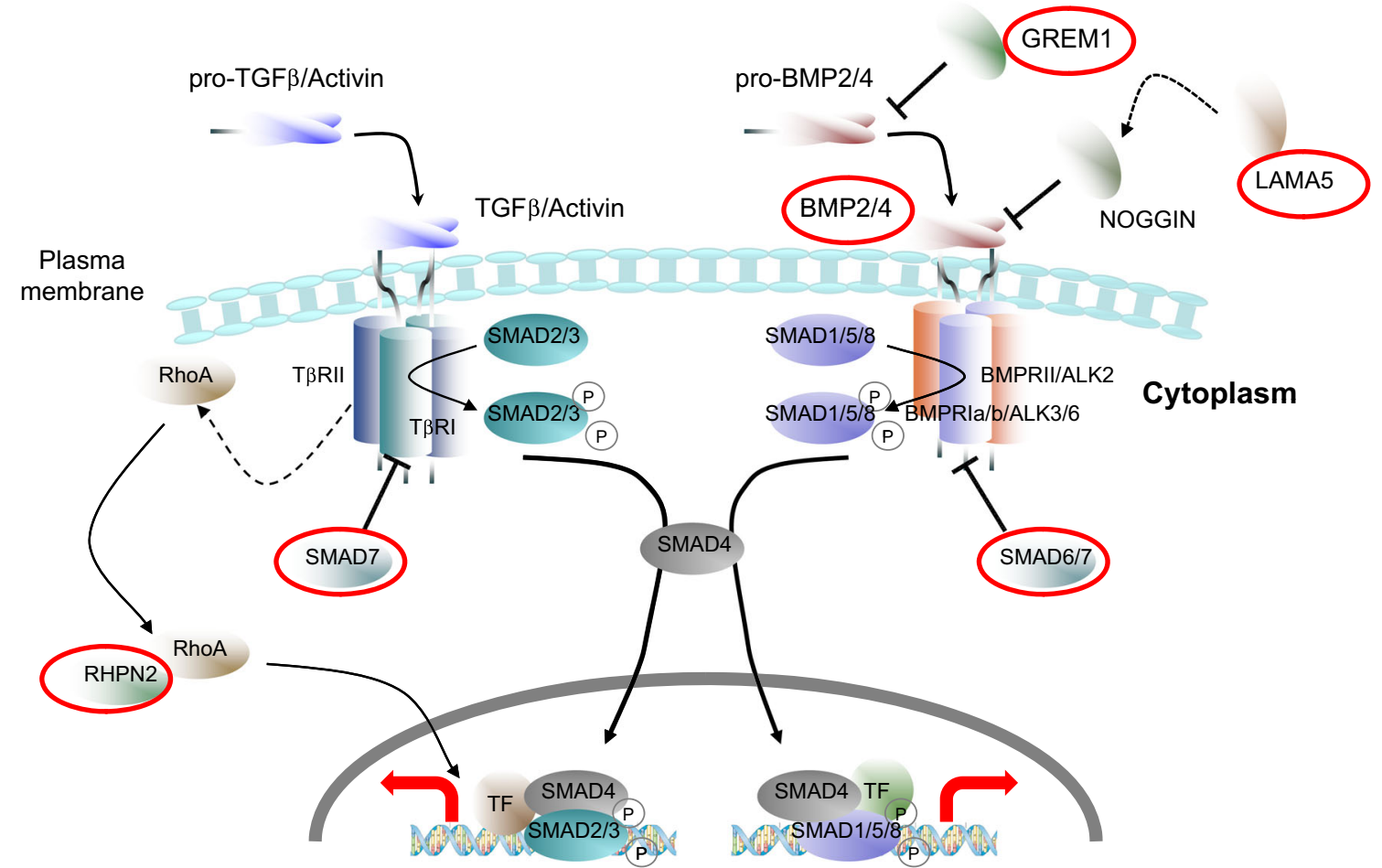

Fig. 1 Genes involved in the transforming growth factor- $\beta$ (TGF $\beta$ ) pathway implicated in colorectal cancer risk by genome-wide association study (GWAS)-identified loci. Six loci either within or near the genes

\section{Nucleus}

circled in red-SMAD7, GREM1, RHPN2, BMP2, BMP4, and LAMA5are implicated as risk factors for colorectal cancer. Figure provided by Graham Casey
Fig. 2 Pleiotropy among different cancers. The riskassociated loci for each cancer are indicated by chromosomal location, and sharing is indicated by colored lines connecting different cancers. CLL chronic lymphocytic leukemia. Reprinted by permission from Macmillan Publishers Ltd: Nature Genetics [78] copyright 2013

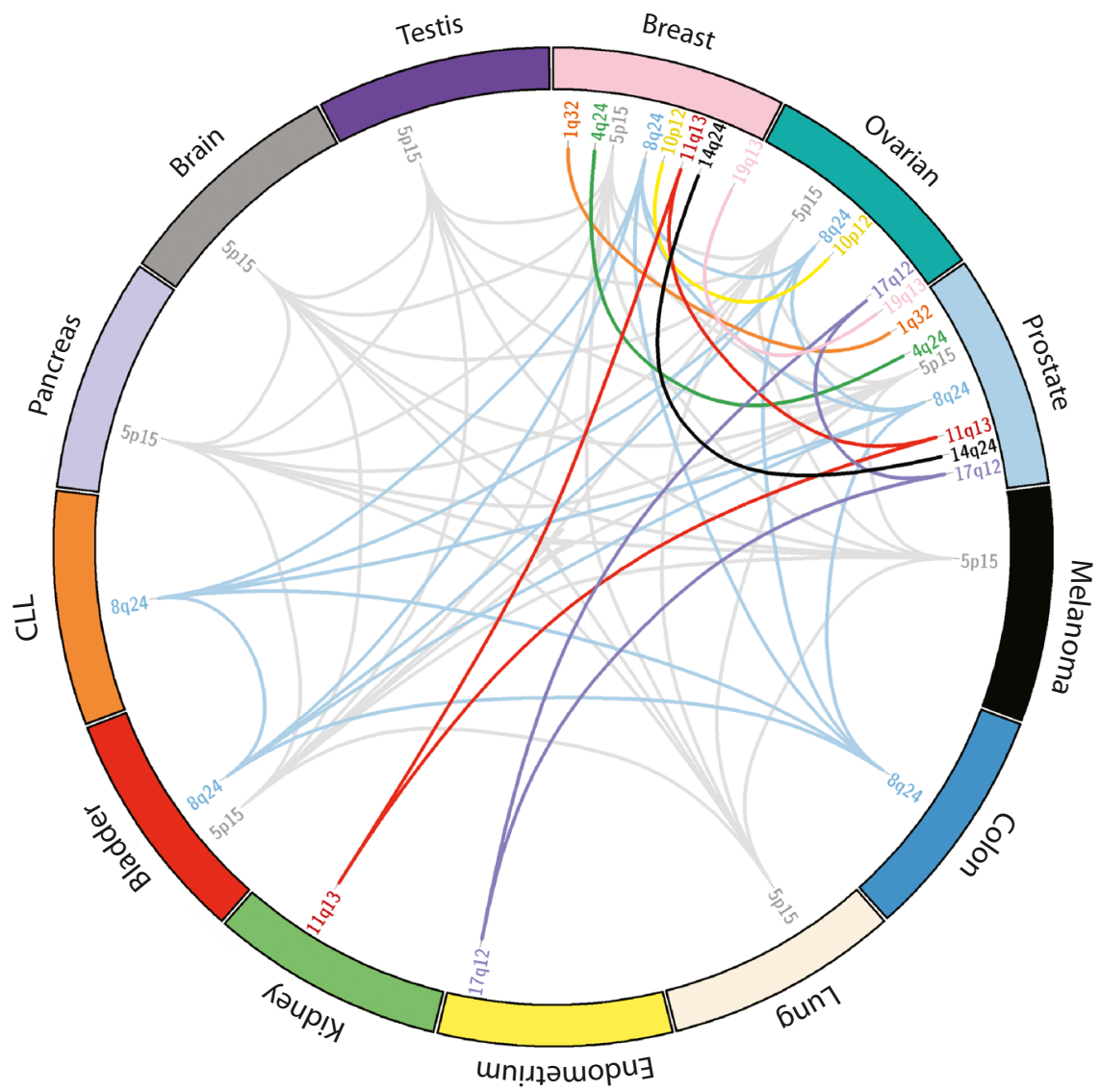


susceptibility to prostate and breast cancers [31]. Pleiotropic associations have also been observed between cancer and noncancer phenotypes - for example, endometrial and prostate cancers and type 2 diabetes at $H N F 1 B$ on 17q12 [32-34], and prostate cancer and cardiovascular disease at the $C D K N 2 B-C D K N 2 A$ gene cluster region at 9p21 $[35,36]$. Thus, GWAS have begun to reveal a shared genetic and biological basis between multiple disease phenotypes, which can be used to discover novel relationships between SNPs, phenotypes, and networks of interrelated phenotypes to provide novel mechanistic insights.

\section{Impact on Public Health and Prevention}

Unlike somatic cancer genetics, where mutated genes highlight potentially actionable drug targets for a select subset of patients, germline risk variants are of low penetrance and are carried by a large fraction of the population that never develop cancer. Thus, a therapeutic prevention or treatment approach of targeting one of the potentially hundreds of risk loci for cancer is not currently feasible or sensible. Consequently, research on the potential clinical utility of GWAS findings has focused on modeling of genetic risk and incorporation of risk stratification into targeted strategies for screening and prevention.

Currently, managing cancer risk in the general population entails both promotion of behaviors that reduce modifiable risk factors and/or routine screening for early detection of lesions with malignant potential. In high-risk populations typically defined by family history, use of selected chemoprevention agents, prophylactic surgery, and/or increased frequency of screening may also be recommended. Given the wide spectrum of lifetime risk in both the average and highrisk populations, it is apparent that additional information is needed beyond family history to tailor recommendations to best balance the risks and benefits associated with different prevention strategies. This raises the question of whether an aggregate set of risk alleles might also improve the effectiveness of currently available prevention strategies through targeting of the appropriate "at risk" population. For the common cancers, this seems encouraging given that the common risk alleles in aggregate can stratify risk 3 - to 6-fold when comparing the top $10 \%$ and $1 \%$, respectively, with the average risk in the population $[5 \bullet, 6 \bullet]$.

\section{Tailoring of Screening Strategies}

There have been several studies and commentaries on the

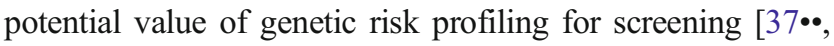
38-41]. In breast cancer, mammographic screening has remained a controversial topic following decades of research, including 10 randomized clinical trials, which showed inconsistent evidence for a beneficial effect on overall mortality [ 42 , 43]. At best, screening appears to have only a small impact in reducing the incidence of advanced cancers [44], with substantive rates of false positives, overdiagnosis, and overtreatment [43, 44]; therefore, tailoring recommendations is a high research priority. Recently, Pashayan et al. [45] evaluated the clinical utility of polygenic risk stratification based on findings from the Collaborative Oncological Gene-environment Study (COGS) in improving the effectiveness and cost effectiveness of screening. Compared with screening women on the basis of age alone (women aged 47-73 years; 10-year absolute risk $\geq 2.5 \%$ ), personalized screening of women aged 35-79 years at the same risk threshold, using a 67-SNP panel, resulted in $24 \%$ fewer women being eligible for screening, while potentially detecting $3 \%$ fewer cases. Such findings are promising; one important issue will be discriminating between subgroups of breast cancer - in particular, estrogen receptornegative $(E R-)$ disease is associated with a poorer prognosis than estrogen receptor-positive $(\mathrm{ER}+)$ disease, and to date there have been only a handful of SNPs specifically associated with ER-disease [46].

In prostate cancer, the value of serum-based prostatespecific antigen (PSA) screening remains controversial. According to randomized clinical trials $[47,48]$, early detection of prostate cancer by screening may prevent death for a subset of men, but the rates of overdiagnosis and overtreatment are substantial, limiting enthusiasm for PSA screening as a population-based prevention strategy. In a study similar to the above example of breast cancer screening, Pashayan et al. [45], using a genetic risk score based on 72 GWAS, identified prostate cancer genetic variants from COGS compared with a hypothetical screening program with eligibility based on age alone (men aged 55-79 years: 10 -year absolute risk of being diagnosed with prostate cancer $\geq 2 \%$ ). They found that with personalized screening for men aged 45-79 years at the same risk threshold, $19 \%$ fewer men would be eligible for screening, at a cost of $4 \%$ fewer potentially screen-detected cases. Whether targeted screening based on risk profiling may reduce overdiagnosis of indolent disease also needs to be examined. Unfortunately, no risk loci have been identified that can differentiate between advanced and non-advanced disease, and so applying a risk stratification scheme for overall prostate cancer to current screening practices may not reveal individuals who are likely to die from prostate cancer. Further studies are needed to search for variants associated with aggressive prostate cancer and to test the implications of adding polygenic risk profiling to guide decision making in screening for prostate cancer.

For other cancers, more studies are needed to evaluate the impact of risk stratification, including colorectal cancer, where colonoscopy with polypectomy has been demonstrated to 
decrease cancer incidence in both average-risk individuals and high-risk individuals $[49,50]$. Currently, the US Preventive Service Task Force recommendations for screening vary only by age and family history, but genomic risk stratification may also be important in further defining individuals in different categories of risk.

\section{Tailoring of Pharmacogenetic Strategies}

GWAS hold much promise in advancing our knowledge of the pharmacogenetics of various agents used in cancer treatment. In breast cancer, tamoxifen has become the most widely used drug in managing ER+breast cancer [51]. Overall risk reductions of $30-80 \%$ for contralateral breast cancer have been reported in women treated with tamoxifen for ER+breast cancer [52-55]. However, considerable variations in efficacy and toxicity have been observed, and there have been several discussions about weighting these risks and benefits according to age, race, and selected breast cancer risk factors [56]. Recently, genome-wide discoveries have yielded a number of variants in biologically relevant genes (e.g., USP7, TMPRSS3, and SMARCA2) affecting tamoxifen sensitivity [57], which, if validated by other studies, may also contribute to further refinement of potential benefits and risks.

In colorectal cancer, considerable evidence from experimental, epidemiologic, and clinical trials demonstrates that aspirin and other nonsteroidal anti-inflammatory drugs (NSAIDs) reduce the risk of colorectal neoplasms [58-62]. Routine use of aspirin/NSAIDs for chemoprevention of colorectal cancer is not currently recommended, because of uncertainty about its risk-benefit profile. Although several mechanisms have been shown to mediate the anti-cancer benefit of aspirin/NSAIDs, emerging data suggest that aspirin/NSAIDs may inhibit WNT/beta-catenin signaling, one of the most essential oncogenic pathways in colorectal cancer [63-65]. Recently, the association between regular use of aspirin and colorectal cancer risk has been shown to differ according to the variation at the colorectal cancer susceptibility locus rs6983267 on chromosome 8q24, which has been functionally linked to WNT/betacatenin signaling activity [66]. In a genome-wide study of 9,000 cases and 9,000 controls, which investigated gene-aspirin interactions, several loci (rs2965667, rs10505806, and rs10283740) in genes involved in WNT signaling were also found to be differentially associated with the beneficial effects of aspirin/NSAID use [67]. Hence, understanding the interplay between genetic markers and aspirin/NSAID use can help to identify subgroups in the population that may preferentially benefit from use of agents for chemoprevention.

\section{Future of Association Studies in the Post-genomic Era}

Several questions remain to be answered in order to assess the impact of GWAS in advancing our understanding of etiology and prevention. Many of these questions have to do with the source of the missing heritability that has not yet been explained by GWAS. At least three possible sources have been much discussed in the literature - namely (1) gene by gene interactions; (2) a larger role for rare variants in common diseases; and (3) an extremely polygenic basis of complex disease.

For the first of these scenarios, it has been argued [68] that such interactions imply that traditional family-based estimates of the additive heritability of many traits or diseases (that portion of heritability that would be directly detectable in single-SNP analyses in GWAS) have been consistently overestimated in earlier (pre-GWAS) work, leading to exaggerated expectations of what can be accomplished in GWAS analyses. The second scenario has been discussed in many contexts; it was noted by Pritchard [69], for example, that even quite mild selection against diseaserelated variants will shift the spectrum of variants that contribute to genetic risk; thus, rare variants contribute much more to total trait or disease heritability than they would under neutral selection. The third scenario is also seriously discussed by many investigators [70-72].

The happiest state of nature from a translational point of view-specifically for risk prediction and screening-based prevention - is if the third scenario dominates. While large sample sizes will be required to identify and calibrate the components of a complex polygenic predictor variable, the third scenario implies that steady progress is expected using today's GWAS platforms (assisted by the imputation of unmeasured variants), so long as sample sizes in conventional GWAS continue to increase. It also implies that today's GWAS data will remain valuable in translating results to patients in the future, since the variants that are identified in these very large studies will be imputable on the basis of the SNPs that have already been genotyped, i.e., the polygene would be expected to be well predicted using today's technology. The second scenario (rare variants) may require much more expensive methods (e.g., whole-genome sequencing) to identify the risk variants, and these variants may be very poorly imputed on the basis of today's GWAS data, so the whole enterprise of detecting, estimating effects, developing a risk model, and applying these risk models to individuals will be much more expensive in the second scenario than in the third scenario. This is further compounded by the observation that rare variants tend to be population specific, so good results in one population would likely not translate to good results in other populations. Even these obstacles are not as great, however, as those expected in the first scenario, where the number of comparisons required to identify complex interactions are vastly greater than currently conceivable sample sizes are likely to support. 
Arguments against either of these first two scenarios playing dominating roles in the missing heritability puzzle for most complex diseases are provided by several lines of evidence. First, since rare SNPs are generally confined to specific racial/ethnic populations, the findings that many associations with common SNPs detectable in one racial/ethnic population are also reproducible in other different populations speaks against one of the corollaries of the rare-variant hypothesis [73], which is that many associations that are observed with common variants are "synthetic" associations with rare variants at the bottom of them. The general consistency of single-SNP associations over different populations also argues against a dominant role for gene $\times$ gene interactions, since single-SNP associations, which are manifestations of interactions between two (or more) alleles, are sensitive to the allele frequencies of both (which tend to vary with the population). Another line of evidence against both of the first two scenarios is provided by the many GWAS-based estimates of complex trait heritability, which have been calculated using the variance components methods of Yang, Visscher, and their colleagues [74-76]. These methods often provide much higher estimates of additive trait heritability due to the common variants measured in GWAS than is evident on the basis of the current set of known hits. While this is encouraging to believers in the GWAS approach, the results of the variance components methods may also be implying that complex trait heritability is dependent upon an extremely large number of common variants, so almost every portion of the genome harbors some variants that affect a given complex trait (of course with very small effect sizes).

In any event, it seems a certainty that additional progress in unearthing missing heritability will require extremely large sample sizes [77]. This is true whether it is rare variants with large effects or common variants with very small effects, and it is especially true if gene-gene interactions are the principal culprits.

\section{Conclusions}

GWAS have made an important leap forward in our understanding of cancer biology by reinforcing the role of known cancer pathways and revealing the unknown biological significance of non-coding regions of the genome that now appear to have important effects on nearby genes, as well as at long distance. On the other hand, GWAS have arguably not identified actionable drug targets, although significant functional exploration has yet to be conducted on most of the loci that may in fact open up new pathways. It is likely that incorporating polygenic risk stratification into tailoring of prevention strategies may prove to be of great clinical utility, at least for some cancers. Critically important will be discrimination of individuals with greater potential for aggressive subtypes of cancers, which may merit enriched screening and other approaches. Overall, the advantages of personalized screening include improving the efficiency of screening programs, detecting cancer in younger individuals, who tend to have more aggressive forms of the disease, and reducing harms from false positive findings through screening of fewer individuals. With polygenic profiling and risk stratification, a subgroup of the population at low risk of cancer may receive screening at lower frequency. Such tailoring of screening strategies to different risk groups may in turn improve the balance between the benefits and harms of screening. Clearly, implementation of a risk-stratified screening program in a population is much more complex than implementation of a program with eligibility based on age or family history alone. The issue of how to develop a dynamic risk score that incorporates technological advances of the rapidly evolving field of genomics and changes over time in individual's non-genetic risk factors will also involve further research. Lastly, the cost effectiveness of genome-based risk profiling will also need to be explored in addition to the optimal timing and frequency of use, ethical considerations, and public approval.

\section{Compliance with Ethics Guidelines}

Conflict of Interest J.C. Figueiredo, D.O. Stram, and C.A. Haiman all declare no conflicts of interest.

Human and Animal Rights and Informed Consent This article does not contain any studies with human or animal subjects performed by any of the authors.

\section{References}

Papers of particular interest, published recently, have been highlighted as:

- Of importance

•- Of major importance

1. Zanke BW, Greenwood CM, Rangrej J, et al. Genome-wide association scan identifies a colorectal cancer susceptibility locus on chromosome 8q24. Nat Genet. 2007;39(8):989-94.

2. Yeager M, Orr N, Hayes RB, et al. Genome-wide association study of prostate cancer identifies a second risk locus at 8q24. Nat Genet. 2007;39(5):645-9.

3. Easton DF, Pooley KA, Dunning AM, et al. Genome-wide association study identifies novel breast cancer susceptibility loci. Nature. 2007;447(7148):1087-93.

4. Hindorff LA, MacArthur J, Morales J, et al. A catalog of published genome-wide association studies. http://www.genome.gov/ gwastudies.

5. Michailidou K, Hall P, Gonzalez-Neira A, et al. Large-scale genotyping identifies 41 new loci associated with breast cancer risk. Nature Genetics. 2013;45(4):353-361, 361e1-2. Recent meta-analysis of nine genome-wide association studies of women 
of European ancestry, which identified 41 new breast cancer susceptibility loci.

6. Eeles RA, Olama AA, Benlloch S, et al. Identification of 23 new prostate cancer susceptibility loci using the iCOGS custom genotyping array. Nature Genetics. 2013;45(4):385-391, 391e1-2. Recent meta-analysis from the international PRACTICAL Consortium, which identified 23 new prostate cancer susceptibility loci.

7. Manolio TA, Collins FS, Cox NJ, et al. Finding the missing heritability of complex diseases. Nature. 2009;461(7265):747-53. Discussion of the potential sources of missing heritability in complex diseases and research strategies that could help illuminate our understanding of the genetics to enable effective disease prevention or treatment.

8.• Consortium EP, Birney E, Stamatoyannopoulos JA, et al. Identification and analysis of functional elements in $1 \%$ of the human genome by the ENCODE Pilot Project. Nature. 2007;447(7146):799-816.

9. Rhie SK, Coetzee SG, Noushmehr H, et al. Comprehensive functional annotation of seventy-one breast cancer risk loci. PLoS One. 2013;8(5):e63925.

10. Hazelett DJ, Rhie SK, Gaddis M, et al. Comprehensive functional annotation of 77 prostate cancer risk loci. PLoS Genet. 2014;10(1): e1004102.

11. Xiang JF, Yin QF, Chen T, et al. Human colorectal cancer-specific CCAT1-L lncRNA regulates long-range chromatin interactions at the MYC locus. Cell Res. 2014;24(5):513-31.

12. Yang $\mathrm{L}$, Lin $\mathrm{C}$, Jin $\mathrm{C}$, et al. IncRNA-dependent mechanisms of androgen-receptor-regulated gene activation programs. Nature. 2013;500(7464):598-602.

13. Massague J, Seoane J, Wotton D. SMAD transcription factors. Genes Dev. 2005;19(23):2783-810.

14. Massague J. TGFbeta in cancer. Cell. 2008;134(2):215-30.

15. Houlston RS, Webb E, Broderick P, et al. Meta-analysis of genomewide association data identifies four new susceptibility loci for colorectal cancer. Nat Genet. 2008;40(12):1426-35.

16. Broderick P, Carvajal-Carmona L, Pittman AM, et al. A genome-wide association study shows that common alleles of SMAD7 influence colorectal cancer risk. Nat Genet. 2007;39(11):1315-7.

17. Jaeger E, Webb E, Howarth K, et al. Common genetic variants at the CRAC1 (HMPS) locus on chromosome 15q13.3 influence colorectal cancer risk. Nat Genet. 2008;40(1):26-8.

18. Peters U, Hutter CM, Hsu L, et al. Meta-analysis of new genomewide association studies of colorectal cancer risk. Hum Genet. 2012;131(2):217-34.

19. Houlston RS, Cheadle J, Dobbins SE, et al. Meta-analysis of three genome-wide association studies identifies susceptibility loci for colorectal cancer at 1q41,3q26.2, 12q13.13 and 20q13.33. Nat Genet. 2010;42(11):973-7.

20. Ghoussaini M, Pharoah PD, Easton DF. Inherited genetic susceptibility to breast cancer: the beginning of the end or the end of the beginning? Am J Pathol. 2013;183(4):1038-51.

21. Lilja H, Abrahamsson PA. Three predominant proteins secreted by the human prostate gland. Prostate. 1988;12(1):29-38.

22. Garde SV, Basrur VS, Li L, et al. Prostate secretory protein (PSP94) suppresses the growth of androgen-independent prostate cancer cell line (PC3) and xenografts by inducing apoptosis. Prostate. 1999;38(2):118-25.

23. Grisanzio C, Werner L, Takeda D, et al. Genetic and functional analyses implicate the NUDT11, HNF1B, and SLC22A3 genes in prostate cancer pathogenesis. Proc Natl Acad Sci U S A. 2012;109(28):11252-7.

24. Waters KM, Stram DO, Le Marchand L, et al. A common prostate cancer risk variant $5^{\prime}$ of microseminoprotein-beta (MSMB) is a strong predictor of circulating beta-microseminoprotein (MSP) levels in multiple populations. Cancer Epidemiol Biomark Prev. 2010;19(10):2639-46.

25. Haiman CA, Stram DO, Vickers AJ, et al. Levels of betamicroseminoprotein in blood and risk of prostate cancer in multiple populations. J Natl Cancer Inst. 2013;105(3):237-43.

26. Sivakumaran S, Agakov F, Theodoratou E, et al. Abundant pleiotropy in human complex diseases and traits. Am J Hum Genet. 2011;89(5):607-18.

27. Rothman N, Garcia-Closas M, Chatterjee N, et al. A multi-stage genome-wide association study of bladder cancer identifies multiple susceptibility loci. Nat Genet. 2010;42(11):978-84.

28. Thomas G, Jacobs KB, Yeager M, et al. Multiple loci identified in a genome-wide association study of prostate cancer. Nat Genet. 2008;40(3):310-5.

29. Haiman CA, Chen GK, Vachon CM, et al. A common variant at the TERT-CLPTM1L locus is associated with estrogen receptor-negative breast cancer. Nat Genet. 2011;43(12): $1210-4$.

30. Turnbull C, Rapley EA, Seal S, et al. Variants near DMRT1, TERT and ATF7IP are associated with testicular germ cell cancer. Nat Genet. 2010;42(7):604-7.

31. Song H, Koessler T, Ahmed S, et al. Association study of prostate cancer susceptibility variants with risks of invasive ovarian, breast, and colorectal cancer. Cancer Res. 2008;68(21):8837-42.

32. Gudmundsson J, Sulem P, Gudbjartsson DF, et al. Genome-wide association and replication studies identify four variants associated with prostate cancer susceptibility. Nat Genet. 2009;41(10): $1122-6$.

33. Kasper JS, Liu Y, Giovannucci E. Diabetes mellitus and risk of prostate cancer in the Health Professionals Follow-Up Study. Int J Cancer. 2009; 124(6):1398-403.

34. Spurdle AB, Thompson DJ, Ahmed S, et al. Genome-wide association study identifies a common variant associated with risk of endometrial cancer. Nat Genet. 2011;43(5):451-4.

35. Dichgans M, Malik R, Konig IR, et al. Shared genetic susceptibility to ischemic stroke and coronary artery disease: a genome-wide analysis of common variants. Stroke. 2014;45(1):24-36.

36. Kerns SL, Stone NN, Stock RG, Rath L, Ostrer H, Rosenstein BS A 2-stage genome-wide association study to identify single nucleotide polymorphisms associated with development of urinary symptoms after radiotherapy for prostate cancer. J Urol. 2013;190(1):102-8.

37.• Burton H, Chowdhury S, Dent T, Hall A, Pashayan N, Pharoah P. Public health implications from COGS and potential for risk stratification and screening. Nat Genet. 2013;45(4):349-51. Discussion regarding the potential public health implications of the findings from the Collaborative Oncological Gene-environment Study (COGS).

38. Chowdhury S, Dent T, Pashayan N, et al. Incorporating genomics into breast and prostate cancer screening: assessing the implications. Genet Med. 2013;15(6):423-32.

39. Khoury MJ, Janssens AC, Ransohoff DF. How can polygenic inheritance be used in population screening for common diseases? Genet Med. 2013;15(6):437-43.

40. Pashayan N, Guo Q, Pharoah PD. Personalized screening for cancers: should we consider polygenic profiling? Per Med. 2013;1: 10(6). doi:10.2217/pme.13.46.

41. Pashayan N, Pharoah P. Population-based screening in the era of genomics. Per Med. 2012;9(4):451-5.

42. Nelson HD, Tyne K, Naik A, et al. Screening for breast cancer: an update for the U.S. Preventive Services Task Force. Ann Intern Med. 2009;151(10):727-37. W237-242.

43. Miller AB, Wall C, Baines CJ, Sun P, To T, Narod SA. Twenty five year follow-up for breast cancer incidence and mortality of the Canadian National Breast Screening Study: randomised screening trial. BMJ. 2014;348:g366. 
44. Bleyer A, Welch HG. Effect of three decades of screening mammography on breast-cancer incidence. N Engl J Med. 2012;367(21):1998-2005.

45. Pashayan N, Hall A, Chowdhury S, Dent T, Pharoah PD, Burton H. Public health genomics and personalized prevention: lessons from the COGS project. J Intern Med. 2013;274(5):451-6.

46. Garcia-Closas M, Couch FJ, Lindstrom S, et al. Genome-wide association studies identify four ER negative-specific breast cancer risk loci. Nat Genet. 2013;45(4):392-8. 398e1-2.

47. Andriole GL, Crawford ED, Grubb 3rd RL, et al. Mortality results from a randomized prostate-cancer screening trial. N Engl J Med. 2009;360(13):1310-9.

48. Schroder FH, Hugosson J, Roobol MJ, et al. Screening and prostate-cancer mortality in a randomized European study. N Engl J Med. 2009;360(13):1320-8.

49. Jarvinen HJ, Aarnio M, Mustonen H, et al. Controlled 15-year trial on screening for colorectal cancer in families with hereditary nonpolyposis colorectal cancer. Gastroenterology. 2000;118(5): 829-34.

50. Winawer SJ, Zauber AG, Ho MN, et al. Prevention of colorectal cancer by colonoscopic polypectomy. The National Polyp Study Workgroup. N Engl J Med. 1993;329(27):197781.

51. Jordan VC. Tamoxifen treatment for breast cancer: concept to gold standard. Oncology. 1997;11(2 Suppl 1):7-13.

52. Bertelsen L, Bernstein L, Olsen JH, et al. Effect of systemic adjuvant treatment on risk for contralateral breast cancer in the Women's Environment, Cancer and Radiation Epidemiology Study. J Natl Cancer Inst. 2008;100(1):32-40.

53. Cook LS, Weiss NS, Schwartz SM, et al. Population-based study of tamoxifen therapy and subsequent ovarian, endometrial, and breast cancers. J Natl Cancer Inst. 1995;87(18):1359-64.

54. Gajalakshmi CK, Shanta V, Hakama M. Risk factors for contralateral breast cancer in Chennai (Madras). India Int J Epidemiol. 1998;27(5):743-50.

55. Fisher B, Dignam J, Bryant J, et al. Five versus more than five years of tamoxifen therapy for breast cancer patients with negative lymph nodes and estrogen receptor-positive tumors. J Natl Cancer Inst. 1996;88(21):1529-42.

56. Gail MH, Costantino JP, Bryant J, et al. Weighing the risks and benefits of tamoxifen treatment for preventing breast cancer. J Natl Cancer Inst. 1999;91(21):1829-46.

57. Weng L, Ziliak D, Im HK, et al. Genome-wide discovery of genetic variants affecting tamoxifen sensitivity and their clinical and functional validation. Ann Oncol. 2013;24(7):1867-73.

58. Chan AT, Giovannucci EL, Meyerhardt JA, Schernhammer ES, Curhan GC, Fuchs CS. Long-term use of aspirin and nonsteroidal anti-inflammatory drugs and risk of colorectal cancer. JAMA. 2005;294(8):914-23.

59. Friis S, Poulsen AH, Sorensen HT, et al. Aspirin and other non-steroidal anti-inflammatory drugs and risk of colorectal cancer: a Danish cohort study. Cancer Causes Control. 2009;20(5):731-40.

60. Garcia Rodriguez LA, Huerta-Alvarez C. Reduced incidence of colorectal adenoma among long-term users of nonsteroidal antiinflammatory drugs: a pooled analysis of published studies and a new population-based study. Epidemiology. 2000;11(4): 376-81.

61. La Vecchia C, Negri E, Franceschi S, et al. Aspirin and colorectal cancer. Br J Cancer. 1997;76(5):675-7.

62. Sandler RS, Galanko JC, Murray SC, Helm JF, Woosley JT. Aspirin and nonsteroidal anti-inflammatory agents and risk for colorectal adenomas. Gastroenterology. 1998;114(3):441-7.

63. Bos CL, Kodach LL, van den Brink GR, et al. Effect of aspirin on the WNT/beta-catenin pathway is mediated via protein phosphatase 2A. Oncogene. 2006;25(49):6447-56.

64. Dihlmann S, Siermann A, von Knebel DM. The nonsteroidal antiinflammatory drugs aspirin and indomethacin attenuate betacatenin/TCF-4 signaling. Oncogene. 2001;20(5):645-53.

65. Kishimoto $\mathrm{Y}$, Takata N, Jinnai $\mathrm{T}$, et al. Sulindac and a cyclooxygenase-2 inhibitor, etodolac, increase APC mRNA in the colon of rats treated with azoxymethane. Gut. 2000;47(6): $812-9$.

66. Nan H, Morikawa T, Suuriniemi M, et al. Aspirin use, 8q24 single nucleotide polymorphism rs6983267, and colorectal cancer according to CTNNB1 alterations. J Natl Cancer Inst. 2013;105(24): $1852-61$.

67. Nan H, Hutter CM, Lin Y, et al. Genome-wide gene-environment interaction analysis of aspirin and non-steroidal anti-inflammatory drug use and colorectal cancer identifies loci at chromosomes $12 \mathrm{p} 12.3$ and $15 \mathrm{q} 25.2$. JAMA. In press.

68. Zuk O, Hechter E, Sunyaev SR, Lander ES. The mystery of missing heritability: genetic interactions create phantom heritability. Proc Natl Acad Sci U S A. 2012;109(4):1193-8.

69. Pritchard JK. Are rare variants responsible for susceptibility to complex diseases? Am J Hum Genet. 2001;69(1):124-37.

70. Speed D, Hemani G, Johnson MR, Balding DJ. Improved heritability estimation from genome-wide SNPs. Am J Hum Genet. 2012;91(6):1011-21.

71. Yang J, Weedon MN, Purcell S, et al. Genomic inflation factors under polygenic inheritance. Eur J Hum Genet. 2011;19(7): 807-12.

72. Hill WG. Understanding and using quantitative genetic variation Philos Trans R Soc Lond Ser B Biol Sci. 2010;365(1537):73-85.

73. Dickson SP, Wang K, Krantz I, Hakonarson H, Goldstein DB. Rare variants create synthetic genome-wide associations. PLoS Biol. 2010;8(1):e1000294.

74. Yang J, Benyamin B, McEvoy BP, et al. Common SNPs explain a large proportion of the heritability for human height. Nat Genet. 2010;42(7):565-9.

75. Yang J, Lee SH, Goddard ME, Visscher PM. Genome-wide complex trait analysis (GCTA): methods, data analyses, and interpretations. Methods Mol Biol. 2013;1019:215-36.

76. Yang J, Lee SH, Goddard ME, Visscher PM. GCTA: a tool for genome-wide complex trait analysis. Am J Hum Genet. 2011;88(1): 76-82.

77. Zuk O, Schaffner SF, Samocha K, et al. Searching for missing heritability: designing rare variant association studies. Proc Natl Acad Sci U S A. 2014;111(4):E455-64.

78. Sakoda LC, Jorgenson E, Witte JS. Turning of COGS moves forward findings for hormonally mediated cancers. Nat Genet. 2013;45(4):345-8. 